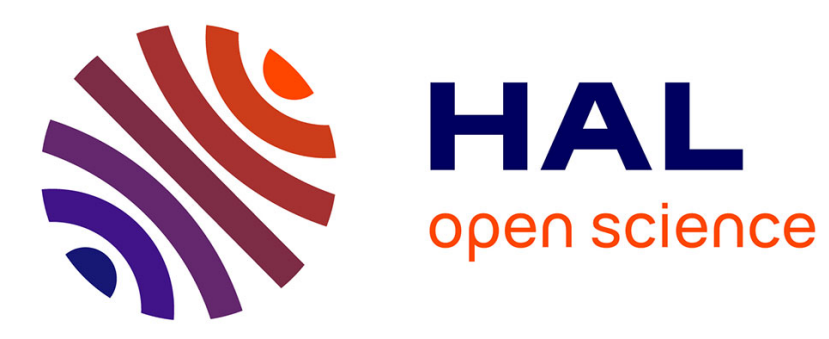

\title{
Analogical Proportions and Multiple-Valued Logics
}

\author{
Henri Prade, Gilles Richard
}

\section{To cite this version:}

Henri Prade, Gilles Richard. Analogical Proportions and Multiple-Valued Logics. 12th European Conference on Symbolic and Quantitative Approaches to Reasoning with Uncertainty (ECSQARU 2013), Jul 2013, Utrecht, Netherlands. pp. 497-509. hal-01197273

\section{HAL Id: hal-01197273 \\ https://hal.science/hal-01197273}

Submitted on 11 Sep 2015

HAL is a multi-disciplinary open access archive for the deposit and dissemination of scientific research documents, whether they are published or not. The documents may come from teaching and research institutions in France or abroad, or from public or private research centers.
L'archive ouverte pluridisciplinaire HAL, est destinée au dépôt et à la diffusion de documents scientifiques de niveau recherche, publiés ou non, émanant des établissements d'enseignement et de recherche français ou étrangers, des laboratoires publics ou privés. 


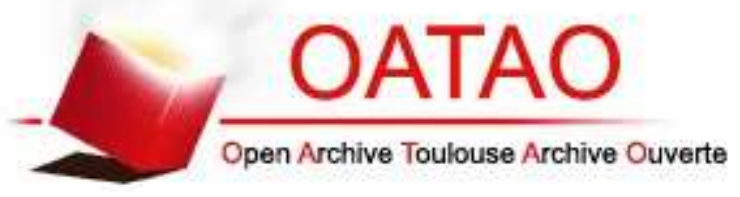

\section{Open Archive TOULOUSE Archive Ouverte (OATAO)}

OATAO is an open access repository that collects the work of Toulouse researchers and makes it freely available over the web where possible.

This is an author-deposited version published in : http://oatao.univ-toulouse.fr/ Eprints ID : 12868

URL: http://dx.doi.org/10.1007/978-3-642-39091-3_42

To cite this version : Prade, Henri and Richard, Gilles Analogical Proportions and Multiple-Valued Logics. (2013) In: 12th European Conference on Symbolic and Quantitative Approaches to Reasoning with Uncertainty (ECSQARU 2013), 7 July 2013 - 10 July 2013 (Utrecht, Netherlands)

Any correspondance concerning this service should be sent to the repository administrator: staff-oatao@listes-diff.inp-toulouse.fr 


\title{
Analogical Proportions and Multiple-Valued Logics
}

\author{
Henri Prade and Gilles Richard \\ IRIT University of Toulouse, 118 rte de Narbonne, Toulouse, France \\ \{prade,richard\}@irit.fr
}

\begin{abstract}
Recently, a propositional logic modeling of analogical proportions, i.e., statements of the form " $A$ is to $B$ as $C$ is to $D$ ", has been proposed, and has then led to introduce new related proportions in a general setting. This framework is well-suited for analogical reasoning and classification tasks about situations described by means of Boolean properties. There is a clear need for extending this approach to deal with the cases where i) properties are gradual ; ii) properties may not apply to some situations ; iii) the truth status of a property is unknown. The paper investigates the appropriate extension in each of these three cases.
\end{abstract}

Keywords: analogical proportion, multiple-valued logic, three-valued logics.

\section{Introduction}

Analogy is not a mere question of similarity between two objects (or situations), but rather a matter of proportion or relation between objects. This view dates back to Aristotle and was enforced by Scholastic philosophy. An analogical proportion equates a relation between two objects with the relation between two other objects. These relations can be considered as a symbolic counterpart to the case where the ratio or the difference between two similar things is a matter of degree or number. As such, an analogical proportion of the form " $A$ is to $B$ as $C$ is to $D$ " poses an analogy of proportionality by (implicitly) stating that the way the two objects $A$ and $B$, otherwise similar, differ is the same way as the two objects $C$ and $D$, which are similar in some respects, differ.

A propositional logic modeling of analogical proportions viewed as a quaternary connective between the Boolean values of some property pertaining to $A$, $B, C$, and $D$ has been proposed in [6]. This logical modeling amounts to precisely state that the difference between $A$ and $B$ is the same as the one between $C$ and $D$, and that the difference between $B$ and $A$ is the same as the one between $D$ and $C$. This view can then be proved to be equivalent to state that the considered Boolean property is true for $A$ and $D$ (resp. $A$ or $D$ ) each time it is true for $B$ and $C$ (resp. $B$ or $C$ ). This latter point shows that a counterpart of a characteristic behavior of numerical geometrical proportions $\left(\frac{a}{b}=\frac{c}{d}\right)$, or of numerical arithmetic proportions $(a-b=c-d)$, namely that the product, or in the second case that the sum, of the extremes is equal to the product (or, in the second case, the sum) of the means, is still observed here. 
The statement of the equality of numerical ratios, or of numerical differences, is useful for extrapolating a fourth value knowing three others that are linked by such a proportionality relation with it. Similarly, the solving of analogical proportion equations is at the basis of an analogical inference process which is of interest for solving non trivial reasoning tasks (e.g., such as IQ tests [2]), or for dealing with classification problems $[5,10]$. The underlying inference mechanism considers four Boolean vectors that describe four situations in terms of $n$ binary properties. When an analogical proportion holds for a large number of properties between the four situations, then one makes the plausible inference that an analogical proportion should also hold for a $(n+1)$ th property whose truth value is known for 3 of the situations, and unknown for the fourth one, which can thus be obtained as a solution of an analogical proportion equation. But, situations may be more generally described in terms of properties that are not always Boolean. This is the case if the properties are gradual, or if they are binary but may not apply. It may also happen that for some situations it is not known if a property holds or not. In these three types of cases (gradual property, property non applicable, and missing information about a property), it is thus of interest to be still able to evaluate in each case if one may consider that an analogical proportion holds. The paper investigates these three cases where different multiple-valued logical calculi are involved.

The paper is organized as follows. After a short background on Boolean analogical proportions (and two related proportions that play a role in the analysis of the problems encountered) in Section 2, the cases of gradual properties, of non-applicable properties and of unknown properties are successively discussed and contrasted in Sections 3, 4, and 5.

\section{Background on Analogical and Related Proportions}

A logical proportion [8] $T(a, b, c, d)$ is a particular type of Boolean expression involving 4 variables $a, b, c, d$, with truth values in $\mathbb{B}=\{0,1\}$. It is made of the conjunction of 2 distinct equivalences, involving a conjunction of variables $a, b$ on one side, and a conjunction of variables $c, d$ on the other side of $\equiv$, where each variable may be negated or not. Both $a \wedge \neg b$ and $\neg a \wedge b$ capture the idea of dissimilarity between $a$ and $b$, while $a \wedge b$ and $\neg a \wedge \neg b$ capture the idea of similarity, positively and negatively. For instance, $(a \bar{b} \equiv c \bar{d}) \wedge(\bar{a} b \equiv \bar{c} d)^{1}$ is the expression of the analogical proportion [6]. As can be seen, analogical proportion uses only dissimilarities and could be informally read as what is true for a and not for $b$ is exactly what is true for $c$ and not for $d$, and vice versa. When a logical proportion does not mix similarities and dissimilarities in its definition, we call it homogeneous: For instance, analogical proportion is homogeneous. More generally, it has been proved that there are 120 semantically distinct logical proportions that can be built. Moreover, each logical proportion has exactly 6 lines leading to 1 in its truth table (and the 10 remaining lines lead to 0 ).

${ }^{1}$ For sake of brevity, $\bar{a}$ is a compact notation for $\neg a$ and $a \bar{b}$ for $a \wedge \neg b$, when useful. 
Two properties seem essential for defining the logical proportions that could be considered as the best counterparts to numerical proportions:

- When all the items are identical, the logical proportion should hold true, i.e., the postulate $T(a, a, a, a)$ should be satisfied.

- The validity of a numerical proportion does not depend on the representation of the numbers in a particular basis. In the same spirit, logical proportions should satisfy the so-called code independency property: $T(a, b, c, d) \Longrightarrow$ $T(\bar{a}, \bar{b}, \bar{c}, \bar{d})$ insuring that the proportion $T$ holds whether we encode falsity as 0 (resp. truth as 1 ) or vice versa.

Only 3 among the 120 proportions satisfy the two previous properties [9]. They are shown in Table 1. They are all homogeneous.

Table 1. 3 remarkable logical proportions: $A, R, P$

\begin{tabular}{|c||c||c|}
\hline $\mathbf{A}$ & $\mathbf{R}$ & $\mathbf{P}$ \\
\hline$a \bar{b} \equiv c \bar{d} \wedge \bar{a} b \equiv \bar{c} d$ & $a \bar{b} \equiv \bar{c} d \wedge \bar{a} b \equiv c \bar{d}$ & $a b \equiv c d \wedge \bar{a} \bar{b} \equiv \bar{c} \bar{d}$ \\
\hline
\end{tabular}

Their truth tables (restricted to the 6 valuations leading to truth value 1 ), are derived from their Boolean expressions, and shown in Table 2.

Table 2. A, R, P: Boolean truth tables

\begin{tabular}{|c|c|c|}
\hline A & $\mathbf{R}$ & $\mathbf{P}$ \\
\hline 0000 & 0000 & 0000 \\
\hline 1111 & 1111 & 1111 \\
\hline 0011 & $\begin{array}{llll}0 & 0 & 1 & 1\end{array}$ & $\begin{array}{llll}1 & 0 & 0 & 1\end{array}$ \\
\hline 1100 & 1100 & 01110 \\
\hline 0101 & 0110 & $\begin{array}{llll}0 & 1 & 0 & 1\end{array}$ \\
\hline 1010 & 1001 & 1010 \\
\hline
\end{tabular}

$A(a, b, c, d)$ is the analogical proportion, which expresses that $a$ (resp. $b$ ) differs from $b$ (resp. $a$ ) as $c$ (resp. $d$ ) differs from $d$ (resp. $c$ ). $R(a, b, c, d)$ is the reverse analogical proportion, where $R(a, b, c, d)=A(a, b, d, c)$ ( $a$ is to $b$ as $d$ is to $c)$. $P(a, b, c, d)$ has been named paralogy [8] and expresses that what $a$ and $b$ have in common, $c$ and $d$ have it also. Most of the semantical properties of these 3 proportions can be easily checked from their truth tables, and may be viewed as counterparts of properties of the numerical (geometrical) proportion $\frac{a}{b}=\frac{c}{d}$. For instance, the property $\frac{a}{b}=\frac{\frac{1}{b}}{\frac{1}{a}}$ parallels the property $T(a, b, \bar{b}, \bar{a})$ (called exchange mirroring) for a logical proportion $T$ where the negation operator plays the role of the inverse. Table 3 summarizes the results: the third column enumerates the proportions among $A, R, P$ satisfying the property respectively named and described in the 1 st and 2 nd columns. Note that $A, R$ and $P$ satisfy the symmetry property $T(a, b, c, d)=T(c, d, a, b)$ : the pairs $(a, b)$ and $(c, d)$ play symmetrical roles. The 2 last lines of Table 3 highlight the strong link between $A, R, P$. Indeed, there also exists an equivalent expression for $A$ that does not involve 
Table 3. Boolean properties of $A, R, P$

\begin{tabular}{|c|c|c|}
\hline Property name & Formal definition & Proportion \\
\hline full identity & $T(a, a, a, a)$ & $\mathrm{A}, \mathrm{R}, \mathrm{P}$ \\
\hline 1-full identity & $T(1,1,1,1) \wedge \neg(0,0,0,0)$ & none \\
\hline 0-full identity & $T(0,0,0,0) \wedge \neg T(1,1,1,1)$ & none \\
\hline reflexivity & $T(a, b, a, b)$ & $\mathrm{A}, \mathrm{P}$ \\
\hline reverse reflexivity & $T(a, b, b, a)$ & $\mathrm{R}, \mathrm{P}$ \\
\hline sameness & $T(a, a, b, b)$ & $\mathrm{A}, \mathrm{R}$ \\
\hline symmetry & $T(a, b, c, d) \rightarrow T(c, d, a, b)$ & $\mathrm{A}, \mathrm{R}, \mathrm{P}$ \\
\hline permutation of means & $T(a, b, c, d) \rightarrow T(a, c, b, d)$ & $\mathrm{A}$ \\
\hline permutation of extremes & $T(a, b, c, d) \rightarrow T(d, b, c, a)$ & $\mathrm{A}$ \\
\hline all permutations of 2 terms & $\forall i, j, T(a, b, c, d) \rightarrow T\left(p_{i, j}(a, b, c, d)\right)$ & none \\
\hline transitivity & $T(a, b, c, d) \wedge T(c, d, e, f) \rightarrow T(a, b, e, f)$ & $\mathrm{A}, \mathrm{P}$ \\
\hline semi-mirroring & $T(a, b, \bar{a}, \bar{b})$ & $\mathrm{R}$ \\
\hline exchange mirroring & $T(a, b, \bar{b}, \bar{a})$ & $\mathrm{A}$ \\
\hline negation compatib. & $T(a, \bar{a}, b, \bar{b})$ & $\mathrm{P}$ \\
\hline link A R & $A(a, b, c, d) \equiv R(a, b, d, c)$ & \\
\hline link A P & $A(a, b, c, d) \equiv P(a, d, c, b)$ & \\
\hline
\end{tabular}

any negation, namely $A(a, b, c, d)=(a \wedge d \equiv b \wedge c) \wedge(a \vee d \equiv b \vee c)$. It looks like the counterpart of the equality of the product of the extremes and of the product of the means for geometrical numerical proportions. As can be seen from this table, the three proportions $A, R, P$, and in particular the analogical proportion $A$, enjoy properties that parallel properties of numerical proportions.

The idea of proportion is closely related to the idea of extrapolation, i.e. to guess / compute a new value on the ground of existing values. In other words, if for some reason, it is believed or known that a proportion should hold between 4 binary items, 3 of them being known, then one may try to infer the value of the 4 th one, at least in the case this extrapolation leads to a unique value. For a proportion $T$, there are exactly 6 distinct valuations for $(a, b, c, d)$ such that $T(a, b, c, d)=1^{2}$. In our context, the problem can be stated as follows. Given a logical proportion $T$ and a 3 -tuple $(a, b, c)$, does it exist a Boolean value $x$ such that $T(a, b, c, x)=1$, and in that case, is this value unique? It is easy to see that there are always cases where the equation has no solution, since the triple $a, b, c$ may take $2^{3}=8$ values, while any proportion $T$ is true only for 6 distinct valuations. For instance, when we deal with analogy $A$, the equations $A(1,0,0, x)$ and $A(0,1,1, x)$ have no solution. And it can be checked that the analogical equation $A(a, b, c, x)$ is solvable iff $(a \equiv b) \vee(a \equiv c)$ holds. In that case, the unique solution is $x=a \equiv(b \equiv c)$. Similar results hold for $R$ and $P$.

$A, R, P$ proportions lead to successful applications when applied to reasoning and classification tasks. To cope with real world applications where objects cannot be simply encoded with a unique Boolean value, we need to extend to Boolean vectors what has been done for a single Boolean value. For a given proportion $T$, the extension to vectors in $\mathbb{B}^{n}$ is done componentwise as follows:

${ }^{2}$ By abuse of notation, we use the same symbol for a variable and its valuation. 


$$
T(\vec{a}, \vec{b}, \vec{c}, \vec{d}) \text { iff } \forall i \in[1, n], T\left(a_{i}, b_{i}, c_{i}, d_{i}\right)
$$

where $\vec{a}=\left(a_{1}, \cdots, a_{n}\right)$ and so on. All the previous properties still hold for $A, R, P$ extensions and the equation solving process, when successful, provides a complete Boolean vector instead of a unique Boolean value. In practice, the analogical inference machinery is then based on the idea that if the same logical proportion holds for a number of components of $\vec{a}, \vec{b}, \vec{c}, \vec{d}$, then it may still hold for a new component known for $\vec{a}, \vec{b}, \vec{c}$, but not for $\vec{d}$, which can then be extrapolated (see e.g., [8]).

However, this vectorial extension may not still be enough for handling practical problems where we have to deal with missing information or properties whose satisfaction is a matter of levels. To cover such situations, extensions of the Boolean interpretation to multiple-valued logics (3-valued at least) is necessary. At this stage, two questions arise:

1 ) in a given model, what are the valuations that correspond to a "perfect" proportion of a given type (i.e., having 1 as truth value)? For instance, does $T(a, a, a, a)$ postulate still have to be satisfied by $A, R, P$ or can we consider models where $A(u, u, u, u)=u, u$ being a truth value distinct from 0 and 1 ?

2) are there valuations that could be regarded as "approximate" proportions (i.e. with a truth value distinct from 0 and 1) of a given type and in that case, what is their truth value?

In order to properly answer these two types of questions, we should carefully distinguish between three cases:

- when property satisfaction is a matter of levels or degrees instead of being binary, i.e. the truth value of a given property may be an intermediary value between 0 and 1 .

- when property satisfaction does not make sense for a given item, i.e., the property is non applicable to it.

- when information about some properties is missing, i.e., we have no clue about the truth value of some properties for some items.

These are the questions we investigate in the following sections keeping in mind an essential principle: the Boolean model should be the limit case of our models when restricted to Boolean valuations.

\section{$3 \quad$ Gradual Properties}

When the satisfaction of properties may be a matter of degree, we have to consider that the truth values belong to a linearly ordered scale $\mathcal{L}$. The simplest case is when $\mathcal{L}=\{0, \alpha, 1\}$, with the ordering $0<\alpha<1$, which can be generalized into a finite chain $\mathcal{L}=\left\{\alpha_{0}=0, \alpha_{1}, \cdots, \alpha_{n}=1\right\}$ or ordered grades $0<\alpha_{1}<$ $\cdots<1$, or to an infinite chain using the real interval [0,1]. A proposal for extending $A$ in such cases has been advocated in [7]. It takes its source in the expression $A(a, b, c, d)=(a \wedge \neg b \equiv c \wedge \neg d) \wedge(\neg a \wedge b \equiv \neg c \wedge d)$, where now 
- i) the central $\wedge$ is taken as equal to min;

- ii) $s \equiv t$ is taken as $\min \left(s \rightarrow_{L} t, t \rightarrow_{L} s\right)$ where $\rightarrow_{L}$ is Eukasiewicz implication, defined by $s \rightarrow_{L} t=\min (1,1-s+t$ ), for $\mathcal{L}=[0,1]$ (in the discrete cases, we take $\alpha=1 / 2$ and $\left.\alpha_{i}=i / n\right)$, and thus $s \equiv t=1-|s-t|$;

- iii) $s \wedge \neg t=\max (0, s-t)=1-\left(s \rightarrow_{L} t\right)$, i.e. $\wedge \neg$ is understood as expressing a bounded difference.

The resulting expression for $A(a, b, c, d)$ is given in Table 4 . Then, we understand the truth value of $A(a, b, c, d)$ as the extent to which the truth values $a, b, c, d$ make an analogical proportion. For instance, in such a graded model, the truth value of $A(0.9,1,1,1)=0.9$, which fits the intuition. It can be checked that the semantics of $A(a, b, c, d)$ thus defined in the graded case, reduces to the previous definition when restricted to the Boolean case. It is interesting to study in what cases $A(a, b, c, d)=1$ and in what cases $A(a, b, c, d)=0$. Then it is clear that $A(a, b, c, d)=1$ when $a-b=c-d$. When $a, b, c, d \in\{0, \alpha, 1\}$ with $\alpha=1 / 2$, it yields the 19 following patterns $(1,1,1,1) ;(0,0,0,0) ;(\alpha, \alpha, \alpha, \alpha) ;(1,0,1,0)$; $(0,1,0,1) ;(1, \alpha, 1, \alpha) ;(\alpha, 1, \alpha, 1) ;(0, \alpha, 0, \alpha) ;(\alpha, 0, \alpha, 0) ;(1,1,0,0) ;(0,0,1,1) ;$ $(1,1, \alpha, \alpha) ;(\alpha, \alpha, 1,1) ;(\alpha, \alpha, 0,0) ;(0,0, \alpha, \alpha) ;(1, \alpha, \alpha, 0) ;(0, \alpha, \alpha, 1) ;(\alpha, 1,0, \alpha)$; $(\alpha, 0,1, \alpha)$.

This means that $A(a, b, c, d)=1$ when the change from $a$ to $b$ has the same direction and the same intensity as the change from $c$ to $d$. However, the last 4 patterns show that there is no need to have $a=b$ and $a=c$ while these conditions hold for the 15 first patterns, which are all of the form $(x, y, x, y)$, $(x, x, y, y)$, or $(x, x, x, x)$. In contrast, note that the last 4 patterns exhibit 3 distinct values.

Table 4. Graded definitions for $A, R, P$ proposed in [7]

\begin{tabular}{|l|}
$A(a, b, c, d)=$ \\
$1-|(a-b)-(c-d)|$ if $a \geq b$ and $c \geq d$, or $a \leq b$ and $c \leq d$ \\
$1-\max (|a-b|,|c-d|)$ if $a \leq b$ and $c \geq d$, or $a \geq b$ and $c \leq d$ \\
\hline$R(a, b, c, d)=A(a, b, d, c)$ \\
\hline$P(a, b, c, d)=$ \\
$\min (1-|\max (a, b)-\max (c, d)|, 1-|\min (a, b)-\min (c, d)|)$ \\
\hline
\end{tabular}

$A(a, b, c, d)=0$ when $a-b=1$ and $c \leq d$, or $b-a=1$ and $d \leq c$, or $a \leq b$ and $c-d=1$, or $b \leq a$ and $d-c=1$. It means the 22 following patterns in the 3 -valued case: $(1,1,1,0) ;(1,1,0,1) ;(1,0,1,1) ;(0,1,1,1) ;(0,0,0,1) ;(0,0,1,0) ;(0,1,0,0)$; $(1,0,0,0) ;(1,0,0,1) ;(0,1,1,0) ;(1,0, \alpha, \alpha) ;(0,1, \alpha, \alpha) ;(\alpha, \alpha, 1,0) ;(\alpha, \alpha, 0,1) ;$ $(1,0,0, \alpha) ;(0,1,1, \alpha) ;(1,0, \alpha, 1) ;(\alpha, 0,0,1) ;(0, \alpha, 1,0) ;(1, \alpha, 0,1) ;(0,1, \alpha, 0)$; $(\alpha, 1,1,0)$. Thus, $A(a, b, c, d)=0$ when the change inside the pairs $(a, b)$ and $(c, d)$ is maximal, while the other pair shows no change or a change in the opposite direction. Thus, $A(a, b, c, d)=\alpha$ for $81-19-22=40$ distinct patterns when we use $\mathcal{L}=\{0, \alpha, 1\}$.

In [7], $R(a, b, c, d)$ is defined by permuting $c$ and $d$ in the definition of $A$, but $P$ is no longer obtained by permuting $b$ and $d$ in the definition of $A$. In fact, $P(a, b, c, d)$ is defined directly from its definition given in Table 1 , changing 
$\neg a \wedge \neg b \equiv \neg c \wedge \neg d$ into $a \vee b \equiv c \vee d$, and taking $\wedge=\min , \vee=\max$, and $s \equiv t=1-|s-t|$, we obtain the definition in Table 4 . If we exchange $b$ and $d$ in this definition, we obtain an alternative definition for the graded analogical proportion, namely

$$
A^{*}(a, b, c, d)=\min (1-|\max (a, d)-\max (b, c)|, 1-|\min (a, d)-\min (b, c)|)
$$

This is the direct counterpart of the definition without negation of the analogical proportion in the Boolean case. It can checked that $A^{*}(a, b, c, d)=1$ only for the 15 patterns with at most two distinct values for which $A(a, b, c, d)=1$, while $A^{*}(a, b, c, d)=\alpha$ for the 4 other patterns for which $A(a, b, c, d)=1$, namely for $(1, \alpha, \alpha, 0) ;(0, \alpha, \alpha, 1) ;(\alpha, 1,0, \alpha) ;(\alpha, 0,1, \alpha)$. Besides, $A^{*}(a, b, c, d)=0$ for only 18 among the 22 patterns that make $A(a, b, c, d)=0$. The 4 patterns for which $A^{*}(a, b, c, d)=\alpha($ instead of 0$)$ are $(1,0, \alpha, \alpha) ;(0,1, \alpha, \alpha) ;(\alpha, \alpha, 1,0) ;(\alpha, \alpha, 0,1)$. Thus, $A^{*}(a, b, c, d)=\alpha$ for $81-15-18=48$ distinct patterns when we use $\mathcal{L}=\{0, \alpha, 1\}$.

Thus, it appears that $A^{*}(a, b, c, d)$ does not acknowledge as perfect the analogical proportion patterns where the amount of change between $a$ and $b$ is the same as between $c$ and $d$ and has the same direction, but where this change applies in different areas of the truth scale. Still, $A^{*}(a, b, c, d)$ remains half-true in these cases, for $\mathcal{L}=\{0, \alpha, 1\}$. When $\mathcal{L}=[0,1]$, it can be checked that $A^{*}(a, b, c, d) \geq 1 / 2$ when $a-b=c-d$; in particular, $\forall a, b, A^{*}(a, b, a, b)=1$, which corresponds to the case where $a=c$ and $b=d$. In the same spirit, if $\mathcal{L}=\{0, \alpha, 1\}$ as well as for $\mathcal{L}=[0,1], A^{*}(a, b, c, d)=0$ when a change inside the pairs $(\mathrm{a}, \mathrm{b})$ and $(\mathrm{c}, \mathrm{d})$ is maximal, while the other pair shows a change in the opposite direction starting from 0 or 1 . However, $A^{*}(1,0, c, c)=\min (c, 1-c)$ and $A^{*}$ takes the same value for the 7 other permutations of $(1,0, c, c)$ obtained by applying symmetry and/or central permutation.

As can be seen in Table $5, A^{*}$ and $A$ also coincide on some patterns having intermediary truth values, but diverge on others. Generally speaking, $A^{*}$ is smoother than $A$ in the sense that more patterns have intermediary truth values with $A^{*}$ than with $A$. $A^{*}$ also maintains the link with $P$, which is no longer true with $A$. However, it would be possible to define another, maybe less natural, graded paralogy as $P^{*}(a, b, c, d)=A(a, d, c, b)$. In practice, the graded version $A$ has been used, apparently in a rather successful way, for classification [10], while $A^{*}$, which is considered here for the first time, has not been experienced yet. It is still unclear if $A^{*}$ may be more suitable for classification purposes.

Table 5. The two graded definitions of the analogical proportion in $[0,1]$

\begin{tabular}{|c|c|}
\hline$A$ & $A^{*}$ \\
$A(1,1, u, v)=1-|u-v|$ & $A^{*}(1,1, u, v)=1-|u-v|$ \\
$A(1,0, u, v)=u-v$ if $u \geq v$ & $A^{*}(1,0, u, v)=\min (u, 1-v)$ \\
$=0$ if $u \leq v$ & \\
$A(0,1, u, v)=v-u$ if $u \leq v$ & $A^{*}(0,1, u, v)=\min (v, 1-u)$ \\
$=0$ if $u \geq v$ & \\
$A(0,0, u, v)=A(1,1, u, v)$ & $A^{*}(0,0, u, v)=A^{*}(1,1, u, v)$ \\
\hline
\end{tabular}


Both $A$ and $A^{*}$ continue to satisfy the symmetry property (as $P, R$, and $P^{*}, R^{*}$ with $\left.R^{*}(a, b, c, d)=A^{*}(a, b, d, c)=P^{*}(a, c, d, b)\right)$. However, only $A^{*}$ still enjoys the means permutation properties and the extremes permutation properties. This is no longer the case with $A$, as shown by the following counter-example. $A(0.8,0.6,1,0.3)=1-|(0.8-0.6)-(1-0.3)|=1-|0.2-0.7|=0.5$ since $0.8 \geq 0.6$ and $1 \geq 0.3$, and $A(0.8,1,0.6,0.3)=1-\max (|0.8-1|,|0.6-0.3|)=$ $1-\max (0.2,0.3)=0.7$ since $0.8 \leq 1$ and $0.6 \geq 0.3$.

But, both $A$ and $A^{*}$ continue to satisfy the code independency property with respect to $\bar{a}=1-a$. Some more Boolean properties which remain valid in the multiple-valued case are summarized in Table 6.

Table 6. Graded properties of $A, A^{*}, R, P$

\begin{tabular}{|c|c|c|}
\hline Property name & Formal definition & Proportion \\
\hline full identity & $T(a, a, a, a)$ & $A^{*}, A, R, P$ \\
\hline reflexivity & $T(a, b, a, b)$ & $A^{*}, A, P$ \\
\hline reverse reflexivity & $T(a, b, b, a)$ & $\mathrm{R}, \mathrm{P}$ \\
\hline sameness & $T(a, a, b, b)$ & $A^{*}, A, R$ \\
\hline symmetry & $T(a, b, c, d) \rightarrow T(c, d, a, b)$ & $A^{*}, A, R, P$ \\
\hline permutation of means & $T(a, b, c, d) \rightarrow T(a, c, b, d)$ & $A^{*}$ \\
\hline permutation of extremes & $T(a, b, c, d) \rightarrow T(d, b, c, a)$ & $A^{*}$ \\
\hline all permutations & $\forall i, j, T(a, b, c, d) \rightarrow T\left(p_{i, j}(a, b, c, d)\right)$ & none \\
\hline semi-mirroring & $T(a, b, \bar{a}, \bar{b})$ & $\mathrm{R}$ \\
\hline exchange mirroring & $T(a, b, \bar{b}, \bar{a})$ & $\mathrm{A}$ \\
\hline negation compatib. & $T(a, \bar{a}, b, \bar{b})$ & none \\
\hline link A R & $A(a, b, c, d) \equiv R(a, b, d, c)$ & \\
\hline link A P & $A(a, b, c, d) \not \equiv P(a, d, c, b)$ & \\
\hline link $A^{*} P$ & $A^{*}(a, b, c, d) \equiv P(a, d, c, b)$ & \\
\hline
\end{tabular}

\section{Non-applicable Properties}

The abbreviation ' $n / a$ ' is currently used in data tables when an attribute does not apply, when a property does not make sense or is not applicable for a particular item. However, the extensive use of ' $\mathrm{n} / \mathrm{a}$ ' may be often ambiguous when it also appears in the same tables when information is not available for some attribute values of some items. Indeed one has to carefully distinguish the case where the property does apply to the item, but it is not known if the property is true or is false for the item, from the case where the property is neither true nor false for the item since the property does not apply to it. The case of unknown truth values is discussed in the next section, while we now address the problem of dealing with genuinely non applicable properties.

The idea of introducing a third truth value for 'not applicable' ( $n a$ for short in the following) in the context of analogy can be already found in the pioneering work of Sheldon Klein $[3,4]$ who was the first to propose to solve analogical proportion equations $A(a, b, c, x)=1$, where $x$ is unknown, as $x=c \equiv(a \equiv b)$ (without providing an explicit definition for $A(a, b, c, d)$ ). However, his handling 
of $n a$ is based on $(n a \equiv n a)=n a$, which suggests that the evaluation of an analogical proportion where $n a$ appears may receive the truth value $n a$, which seems to be more in the spirit of understanding $n a$ as 'not available', or 'unknown'.

Indeed, although a property may be 'true', 'false', or 'not applicable' for an item, it seems natural to expect that $A(a, b, c, d)$ can only be 'true' or 'false', since $(1, n a, 1, n a)$ looks intuitively satisfactory as an analogical proportion, while $(1, n a, 0,0)$ is not. More precisely, the acceptable 4-tuples of valuations that make an analogical proportion true are of the form $(x, x, x, x),(x, y, x, y)$, and $(x, x, y, y)$, where $x, y \in\{0,1, n a\}$, where any other 4-tuple should make it false, since 0,1 and $n a$ play the same role. This leads to acknowledge as true the 15 following patterns $(1,1,1,1) ;(0,0,0,0) ;(n a, n a, n a, n a) ;(1,0,1,0) ;(0,1,0,1)$; $(1, n a, 1, n a) ;(n a, 1, n a, 1) ;(0, n a, 0, n a) ;(n a, 0, n a, 0) ;(1,1,0,0) ;(0,0,1,1)$; $(1,1, n a, n a) ;(n a, n a, 1,1) ;(n a, n a, 0,0) ;(0,0, n a, n a)$, all the others being false.

In other words, we are in a situation somewhat similar to the one encountered in the previous section in the case of a unique intermediary truth-value $\alpha$ between true and false, meaning 'half-true' (or equivalently 'half-false'), when we refuse the four patterns $(1, \alpha, \alpha, 0),(0, \alpha, \alpha, 1),(\alpha, 0,1, \alpha)$ and $(\alpha, 1,0, \alpha)$ as being true, except that now no pattern has the third truth value. It is possible to find logical definitions of the analogical proportion having the expected behavior for the truth values $\{0,1, n a\}$. First, it can be checked that this is obtained with the following expression

$$
A(a, b, c, d)=(a \wedge d \equiv b \wedge c) \wedge(a \vee d \equiv b \vee c)
$$

where the $\{0,1, n a\}$ are ordered as the chain $1>n a>0$ (i.e. $\wedge$ is Kleene conjunction, see, e.g., [1], and $x \equiv y=1$ if and only if $x=y$, and $x \equiv y=0$ otherwise.

A counterpart to $A(a, b, c, d)=(a \backslash b \equiv c \backslash d) \wedge(b \backslash a \equiv d \backslash c)$ where $\backslash$ here denotes the Boolean logical connective corresponding to set difference, can also be found. However, since we do not want to have $(1, n a, n a, 0)$ true, the difference between 1 and $n a$ and the difference between $n a$ and 0 should not be the same, neither the same as between 1 and 0 , nor 1 between 1 for sure. Thus we need 4 distinct values for the difference. This is impossible with 3 truth values! This contrasts with the Boolean case where there are only two possible difference values needed. The solution is then to use 2 connectives for differences:

$x \backslash_{1} y=1$ if $x=1$ and $y=0 ; x \backslash_{1} y=n a$ if $x=1$ and $y=n a ; x \backslash_{1} y=0$ otherwise; $x \backslash_{2} y=1$ if $x=1$ and $y=0 ; x \backslash_{2} y=n a$ if $x=n a$ and $y=0 ; x \backslash_{2} y=0$ otherwise. Then the definition of $A(a, b, c, d)$ becomes

$$
\left(a \backslash_{1} b \equiv c \backslash_{1} d\right) \wedge\left(b \backslash_{2} a \equiv d \backslash_{2} c\right) \wedge\left(a \backslash_{2} b \equiv c \backslash_{2} d\right) \wedge\left(b \backslash_{1} a \equiv d \backslash_{1} c\right)
$$

where $x \equiv y=1$ iff $x=y ; x \equiv y=0$ otherwise; and $\wedge$ is any conjunction connective that coincides with classical conjunction on $\{0,1\}$. This definition yields 1 for the 15 expected patterns and is 0 otherwise for the $81-15=66$ remaining patterns.

It is even possible to find an expression for $A(a, b, c, d)$ where $\backslash_{1}$ and $\backslash_{2}$ are expressed in terms of a conjunction and negations, i.e. where $x \backslash_{1} \mathrm{y}$ is replaced by $x \wedge^{*} \neg_{1}(y)$ and $x \backslash_{2} y$ is replaced by $x \wedge^{*} \neg_{2}(y)$. We obtain a definition for $A(a, b, c, d)$ under the form 
$\left(a \wedge^{*} \neg_{1} b \equiv c \wedge^{*} \neg_{1} d\right) \wedge^{*}\left(b \wedge^{*} \neg_{2} a \equiv d \wedge^{*} \neg_{2} c\right) \wedge^{*}\left(a \wedge^{*} \neg_{2} b \equiv c \wedge^{*} \neg_{2} d\right) \wedge^{*}\left(b \wedge^{*} \neg_{1} a \equiv d \wedge^{*} \neg_{1} c\right)$

where the two negations are Post-like negations defined through a circular ordering of the three truth-values, where the negation of a value is the successor value in the ordering, namely $\neg_{1}(0)=n a ; \neg_{1}(n a)=1 ; \neg_{1}(1)=0$ and $\neg_{2}(0)=1 ; \neg_{2}(n a)=0 ; \neg_{2}(1)=n a$. This acknowledges the fact that in some sense these three truth-values play similar roles. The non-standard three-valued conjunction $\wedge^{*}$, which is defined by

$$
\begin{aligned}
& x \wedge^{*} y=1 \text { if } x=1 \text { and } y=1 \\
& x \wedge^{*} y=n a \text { if } x=n a \text { and } y=n a \\
& x \wedge^{*} y=0 \text { otherwise }
\end{aligned}
$$

also agrees with this view, while coinciding with classical conjunction in the binary case. As in the previous section, we summarize in Table 7 the properties of the Boolean case that remain valid in this 3 -valued model where $n a$, standing for non applicable, is the third truth value.

Table 7. Properties of $A, R, P$ with truth value $n a$ (as non applicable)

\begin{tabular}{|c|c|c|}
\hline Property name & Formal definition & Proportion \\
\hline full identity & $T(a, a, a, a)$ & $\mathrm{A}, \mathrm{R}, \mathrm{P}$ \\
\hline reflexivity & $T(a, b, a, b)$ & $\mathrm{A}, \mathrm{P}$ \\
\hline reverse reflexivity & $T(a, b, b, a)$ & $\mathrm{R}, \mathrm{P}$ \\
\hline sameness & $T(a, a, b, b)$ & $\mathrm{A}, \mathrm{R}$ \\
\hline symmetry & $T(a, b, c, d) \rightarrow T(c, d, a, b)$ & $\mathrm{A}, \mathrm{R}, \mathrm{P}$ \\
\hline permutation of means & $T(a, b, c, d) \rightarrow T(a, c, b, d)$ & $\mathrm{A}$ \\
\hline permutation of extremes & $T(a, b, c, d) \rightarrow T(d, b, c, a)$ & $\mathrm{A}$ \\
\hline all permutations & $\forall i, j, T(a, b, c, d) \rightarrow T\left(p_{i, j}(a, b, c, d)\right)$ & none \\
\hline link A R & $A(a, b, c, d) \equiv R(a, b, d, c)$ & \\
\hline link A P & $A(a, b, c, d) \equiv P(a, d, c, b)$ & \\
\hline
\end{tabular}

\section{Unknown Properties}

In this section, we briefly consider a situation that is quite different from the ones studied in the two previous sections. We assume now that the features used for describing situations are all binary (i.e., they can be only true or false), but their truth value may be unknown. Thus, the possible states of information regarding a Boolean variable $x$ pertaining to a given feature may be represented by one of the 3 truth value subsets $\{0\},\{1\}$ or $\{0,1\}$, corresponding respectively to the case where the truth value of $x$ is false, true or unknown. We denote this state of information by $\tilde{x}$, which is a subset of $\{0,1\}$. The evaluation of a logical proportion $T(a, b, c, d)$ amounts to compute the state of information denoted $\mathcal{T}(\tilde{a}, \tilde{b}, \tilde{c}, \tilde{d})$ about its truth value, knowing $\tilde{a}, \tilde{b}, \tilde{c}, \tilde{d}$. It is given by the standard set extension where $v$ denotes a Boolean valuation:

$$
\mathcal{T}(\tilde{a}, \tilde{b}, \tilde{c}, \tilde{d})=\{v(T(a, b, c, d)) \mid v(a) \in \tilde{a}, v(b) \in \tilde{b}, v(c) \in \tilde{c}, v(d) \in \tilde{d}\}
$$


From now on, we focus on analogical proportion $A$ only, but $R, P$ and $I$ could be handled in a similar manner. For instance, let us take the example $A(a, b, c, d)$ where $\tilde{a}=\{1\}, \tilde{b}=\{0\}, \tilde{c}=\tilde{d}=\{0,1\}$. Applying the previous formula leads to

$$
\mathcal{A}(\tilde{a}, \tilde{b}, \tilde{c}, \tilde{d})=\{0,1\}
$$

since the truth value of $A(a, b, c, d)$ may be 0 for the valuations 1001,1000 , 1011, and 1 for 1010. If we consider the following expression $A(a, b, a, b)$ when $\tilde{a}=\tilde{b}=\{0,1\}$, a similar computation leads to

$$
\mathcal{A}(\tilde{a}, \tilde{b}, \tilde{a}, \tilde{b})=\{1\}
$$

since the truth value of $A(a, b, a, b)$ is 1 for any of the valuations 1010, 1111, 0101, or 0000. Similarly, the truth value of $A(a, a, a, a)$ is 1 , even when $\tilde{a}=\{0,1\}$. But, the set of possible truth values for $A(a, b, c, d)$ is $\{0,1\}$ when $\tilde{a}=\{0,1\}, \tilde{b}=$ $\{0,1\}, \tilde{c}=\{0,1\}, \tilde{d}=\{0,1\}$, i.e. we have the same state of information for all of them. This expresses that the full identity property does not hold any longer at the information level for analogical proportion. And this illustrates the fact that the logic of uncertainty is no longer truth functional, since the state of information about the truth value of $A(a, b, c, d)$ does not only depend on the state of information about the truth values of $a, b, c$, and $d$, but is also constrained by the existence of possible logical dependencies between these variables.

Nevertheless, some key properties of homogeneous proportions remain valid at the information level such as symmetry, or central and extreme permutations. Indeed it can be checked that, for instance, for symmetry:

$$
\mathcal{A}(\tilde{a}, \tilde{b}, \tilde{c}, \tilde{d})=\mathcal{A}(\tilde{c}, \tilde{d}, \tilde{a}, \tilde{b})
$$

Using the set extension evaluation of logical proportions in presence of incomplete information, we can compute the set of possible truth values of the analogical proportion for the different 4-tuples of states of information. We now denote by $u$ the state $\{0,1\}$, and respectively by 0 and 1 , the states of information $\{0\}$ and $\{1\}$. A 4-tuple of states of information will be called information pattern, or pattern for short, and denoted by a 4-tuple of elements of $\{0,1, u\}$ without blank space. For instance, $01 u 1$ is such a pattern and should be understood as the 4-tuple of states of information $(\{0\},\{1\},\{0,1\},\{1\})$.

Then, the 6 patterns 0000,1111,0011,1100,1010,0101 that makes $A$ true in the Boolean case, and where $u$ does not appear, are the only ones that are still true with the above view (for which we get the singleton $\{1\}$ as information state for $A(a, b, c, d))$. As soon as at least one state of information is $u$ in the pattern, the state of information for $A(a, b, c, d)$ is $u$ or 0 . Indeed, for instance, $01 u 0$ leads to 0 since whatever the truth value of the $3 \mathrm{rd}$ variable, the analogical proportion does not hold. Thus, despite the lack of knowledge regarding the $3 \mathrm{rd}$ variable, we know the exact truth value of the proportion in this case, namely it is false. It appears that there are 18 patterns that lead to 0 . They are the 10 patterns of the Boolean case and the 8 following ones: 01u0, $0 u 10, u 001,100 u$, $10 u 1,1 u 01, u 110,011 u$. Thus, in the $81-6-18=57$ remaining cases, the state of information for $A(a, b, c, d)$ is $u$. 
It can be checked that these results can be retrieved both with the initial definition of $A$ or with $A^{*}$ where complete ignorance $u$ is handled with ${ }^{-}, \wedge, \vee$ as the strong Kleene connectives (see [1]) and $\equiv$ as Bochvar connective, where $u$ is an absorbing element. The corresponding truth tables are recalled in Table 8. This provides a way to extend the definition of the analogical proportion in

Table 8. Truth tables for $u$ as lack of knowledge

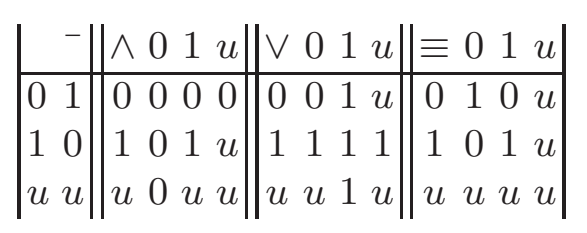

case of lack of knowledge when no dependencies between the variables exist. As in the Boolean case, the definitions $A$ (resp. $R, P, I)$ and $A^{*}\left(\operatorname{resp} . R^{*}, P^{*}, I^{*}\right)$ are equivalent. Nevertheless, this truth-functional calculus provides only a description of the evaluation of the patterns at the information level. Namely, it enables us to retrieve the tri-partition of the patterns in respectively 6, 18 and 57 patterns leading respectively to 1,0 and $u$, but it does not account for the full calculus of the extended definition of logical proportions in presence of incomplete information, when dependencies take place between variables, for instance it can be checked that $A(a, b, a, b)$ and $A^{*}(a, b, a, b)$, when $a$ and $b$ are unknown, does not yield 1 as expected, but $u$ (this is just due to the fact that constraints $a=c$ and $b=d$ are ignored).

\section{Concluding Remarks}

This paper has discussed three extensions of the notion of analogical proportions (and related logical proportions) by carefully distinguishing the problems of handling graded truth values, of dealing with non applicable properties, and of coping with unknown truth values. In each case, a different modeling has been obtained with a different repartition of the patterns found to be true, false, or having another value, and where the set of properties preserved for the analogical proportion is not the same. More generally, it would be of interest of developing an approach where the three types of problem can be handled together.

\section{References}

1. Ciucci, D., Dubois, D.: Relationships between connectives in three-valued logics. In: Greco, S., Bouchon-Meunier, B., Coletti, G., Fedrizzi, M., Matarazzo, B., Yager, R.R. (eds.) IPMU 2012, Part I. CCIS, vol. 297, pp. 633-642. Springer, Heidelberg (2012)

2. Correa, W., Prade, H., Richard, G.: When intelligence is just a matter of copying. In: De Raedt, L., et al. (eds.) Proc. 20th Europ. Conf. on Artificial Intelligence, Montpellier, August 27-31, pp. 276-281. IOS Press (2012) 
3. Klein, S.: Culture, mysticism \& social structure and the calculation of behavior. In: Proc. 5th Eur. Conf. in AI (ECAI 1982), Paris, pp. 141-146 (1982)

4. Klein, S.: Analogy and mysticism and the structure of culture (and Comments \& Reply). Current Anthropology 24(2), 151-180 (1983)

5. Miclet, L., Bayoudh, S., Delhay, A.: Analogical dissimilarity: definition, algorithms and two experiments in machine learning. JAIR 32, 793-824 (2008)

6. Miclet, L., Prade, H.: Handling analogical proportions in classical logic and fuzzy logics settings. In: Sossai, C., Chemello, G. (eds.) ECSQARU 2009. LNCS, vol. 5590, pp. 638-650. Springer, Heidelberg (2009)

7. Prade, H., Richard, G.: Multiple-valued logic interpretations of analogical, reverse analogical, and paralogical proportions. In: Proc. 40th IEEE Inter. Symp. on Multiple-Valued Logic (ISMVL 2010), Barcelona, pp. 258-263 (May 2010)

8. Prade, H., Richard, G.: Reasoning with logical proportions. In: Lin, F.Z., Sattler, U., Truszczynski, M. (eds.) Proc. 12th Inter. Conf. on Principles of Knowledge Representation and Reasoning (KR 2010), Toronto, Ontario, Canada, May 9-13, pp. 545-555. AAAI Press (2010)

9. Prade, H., Richard, G.: Homogeneous logical proportions: Their uniqueness and their role in similarity-based prediction. In: Brewka, G., Eiter, T., McIlraith, S.A. (eds.) Proc. 13th Inter. Conf. on Principles of Knowledge Representation and Reasoning (KR 2012), Roma, June 10-14, pp. 402-412. AAAI Press (2012)

10. Prade, H., Richard, G., Yao, B.: Enforcing regularity by means of analogy-related proportions-a new approach to classification. International Journal of Computer Information Systems and Industrial Management Applications 4, 648-658 (2012) 